\title{
OBTENCION DE COORDENADAS TALAIRACH A PARTIR DE MAPAS CORTICALES DE AREA VISUAL CON RESONANCIA MAGNETICA FUNCIONAL
}

\author{
Drs. Gonzalo Rojas C(1), Jorge Cordovez $M^{(2)}$, Marcelo Gálvez $M^{(2)}$, Takeshi Asahi K(1), Eduardo Bravo C(2). \\ 1. Laboratorio de Imágenes Médicas, Servicio de Neurorradiología, Instituto de Neurocirugía Dr. Asenjo, Santiago, \\ Chile. \\ 2. Servicio de Neurorradiología, Instituto de Neurocirugía Dr. Asenjo, Santiago, Chile.
}

\section{GETTING TALAIRACH COORDINATES FROM THE fMRI MAP- PING OF VISUAL CORTEX}

\begin{abstract}
Functional magnetic resonance imaging gives detailed information on the location of brain activity. Due to the functional-anatomic difference between patients, discrepancies arouse concerning the location of activation areas. To solve this problem, a standard positioning system called Talairach Coordinates was used.

The fMRI mapping of visual cortex was performed in 14 healthy volunteers, using colored circles visual stimulation.

Using fMRI post processing software, a combined image of the 14 volunteers $\mathrm{FMR}$ was computed. The main activation voxel is $(16,-93,7)$, that corresponds to Brodmann area 17 (primary visual area V1). Correlation of the primary visual area (V1) obtained through fMRI with Brodmann area 17, only proven from the classical literary neurophysiological viewpoint, was confirmed.
\end{abstract}

Key words: Brodmann areas, fMRI, FSL, Talairach coordinates, Visual area, V1.

Resumen: La resonancia magnética funcional entrega información detallada sobre la localización de la actividad cerebral. Debido a la diferencia anátomo-funcional entre los pacientes, se producen discrepancias en la localización de las zonas de activación obtenidas. Para solucionar esto, se utiliza un sistema de posicionamiento estándar denominado coordenadas Talairach. Se realizó resonancia magnética funcional con estimulación visual de círculos de colores en 14 voluntarios de ambos sexos. Mediante postproceso con software especializado, se obtuvo

Rojas G, y cols. Obtención de coordenadas Talairach a partir de mapas corticales de área visual con resonancia magnética funcional. Rev Chil Radiol 2008; 14: 57-61.

Correspondencia: Dr. Gonzalo Rojas C.

grojas@computer.org una imagen combinada de los 14 estudios, en la cual el voxel de mayor activación $(16,-93,7)$ corresponde al área de Brodmann 17 (área visual primaria V1). Se confirma la correlación del área funcional visual primaria (V1) obtenida con resonancia magnética funcional y el área 17 de Brodmann, demostrada sólo desde el punto de vista teórico en los textos de neurofisiología clásicos.

Palabras clave: Areas de Brodmann, Area visual, Coordenadas Talairach, fMRI, FSL, V1.

\section{Introducción}

Las imágenes de resonancia magnética cerebral varían de forma importante de un paciente a otro; ésto se debe a diferencias en las características de cada cerebro tales como forma, etapa de desarrollo y tamaño. Por ello, la estandarización es útil para comparar un cerebro con otro, surgiendo el concepto de "normalizar", es decir tomar una muestra significativa de la población normal y obtener un promedio de la morfología cerebral. Este cerebro "normalizado" o promedio se utiliza como patrón de referencia para comparar un cerebro con otro. La normalización se realiza utilizando técnicas de corregistro (traslación, rotación y escalamiento).

Después de la normalización es posible especificar cada ubicación en el cerebro, usando coordenadas esterotáxicas ("Talairach"), que utilizan tres números o puntos en el espacio (X, Y, Z) para describir la distancia a partir de la comisura anterior, origen de este sistema. Estas coordenadas X, Y, Z, varían de izquierda a derecha, posterior-anterior y ventraldorsal y están basadas en el cerebro de Talairach, que es un cerebro disecado y fotografiado en el atlas de Talairach y Tournoux ${ }^{(1,2,3)}$.

El patrón que se utiliza es el denominado MNI152 creado en el Montreal Neurological Institute a partir del atlas MNI305, generado con imágenes de controles sanos (239 hombres, 66 mujeres, edad 23.4 +/-4.1) corregistrados de forma manual y automática al cerebro de Talairach ${ }^{(1)}$. 
El neurólogo alemán Korbinian Brodmann, clasificó en 1909 la corteza cerebral en 52 regiones diferentes basándose en sus características citoarquitectónicas. Estas áreas se llaman actualmente, áreas de Brodmann ${ }^{(4)}$; algunas de éstas fueron posteriormente, asociadas a funciones neurológicas: las áreas 1, 2, y 3 en el giro postcentral del lóbulo parietal ligadas a la región somatosensitiva, las áreas 17 y 18 del lóbulo occipital asociadas a las áreas visuales primarias, entre otras que han sido utilizadas por muchas generaciones de neurólogos y neurocirujanos como patrón de ubicación anátomo-funcional.

La resonancia magnética funcional (fMRI en inglés), es una técnica no invasiva de estudio funcional. Permite la visualización indirecta de ciertas áreas del cerebro que responden a una tarea o estímulo específico. El efecto utilizado, denominado BOLD (Blood Oxygen Level Dependent contrast), mide la respuesta hemodinámica de activación del cerebro. El aumento del flujo sanguíneo focal en el área de activación neuronal local reduce el efecto paramagnético de la deoxihemoglobina causando un aumento en la señal de las imágenes $\mathrm{T} 2$ o $\mathrm{T} 2^{\star(5,6)}$. Esta técnica permite evaluar áreas elocuentes: motora, sensitiva, visual y lenguaje. Para obtener las imágenes se realizan diferentes tests o paradigmas para la estimulación específica de las áreas corticales de interés. Una manera de diseñar los paradigmas es hacerlo con bloques intercalados de tiempos de actividad y tiempos de reposo(6). Se han estudiado extensamente las representaciones de las áreas visuales en la corteza cerebral, utilizando paradigmas de representación de imágenes ${ }^{(6-8)}$.

El objetivo de este trabajo es obtener una imagen de resonancia funcional que indique el área de activación común a partir de la resonancia funcional visual de 14 voluntarios de un estudio publicado previamente ${ }^{(6)}$. Además, se obtendrán las coordenadas de Talairach y las áreas de Brodmann correspondientes a las áreas de activación.

\section{Material y método}

Se aplicó un test específico para la activación del área visual en 14 voluntarios de ambos sexos (7 hombres y 7 mujeres), promedio de edad 33 años, todos diestros. El test aplicado consistió en la proyección de una figura con el aspecto de un espiral de colores en movimiento continuo, por medio de lentes conectados a un sistema de imágenes digitales (Figura 1), alternado con períodos de proyección negra (on-off). Esta proyección se realizó simultáneamente en ambos ojos.

Las fMRI de los 14 voluntarios fueron procesadas de forma individual y sus resultados publicados previamente por Rosales y cols ${ }^{(6)}$. Según los resultados, el análisis estadístico individual (de primer nivel) de las imágenes se realizó con el software FSL v3.3.7
(FMRIB's Software Library, FMRIB Centre, Department of Clinical Neurology, University of Oxford; http:// www.fmrib.ox.ac.uk/fsl/). Los parámetros estadísticos utilizados son de $Z>2.7$ aprox, y un umbral de significación $p=0,05$. Las imágenes se adquirieron en un resonador Phillips Intera 1.5 T. Se utilizaron secuencias FEEPI, TE/TR 50/3000 ms, flip angle $90^{\circ}$, matriz 64x64, FOV 240x240 mm, voxel de $3.7 \times 3.7 \mathrm{~mm}$, espesor de corte $8 \mathrm{~mm}$. Además se adquirió volumen anatómico T1 (3-D-FFE, TE/TR 9.2/25 ms, flip angle $30^{\circ}, 256 \times 256$, FOV 240x240, voxel de $0.94 \times 0.94 \mathrm{~mm}$, y cortes de $1 \mathrm{~mm}$ de espesor) $)^{(6)}$.

El análisis estadístico de alto nivel, es decir la combinación estadística de las fMRI ya procesadas individualmente, se realizó con software FSL v4.0.1 (FMRIB's Software Library, FMRIB Centre, Department of Clinical Neurology, University of Oxford; (http://www. fmrib.ox.ac.uk/fsl), utilizando un modelo de efectos fijos forzando la varianza de efectos aleatorios a 0 in FLAME (FMRIB's Local Analisis of Mixed Effects). El umbral de las imágenes de estadística $Z$ fue fijado utilizando clusters determinados por $Z>2.7$ y un umbral de significación de clusters de $p=0,05$.

A partir de las coordenadas de Talairach de los voxeles de mayor activación se obtuvo el área de Brodmann y la zona neurológica correspondiente mediante el software "Talairach Daemon Client" (Research Imaging Center, University of Texas Health Sciences Center, San Antonio, TX, USA, http://ric. uthscsa.edu).

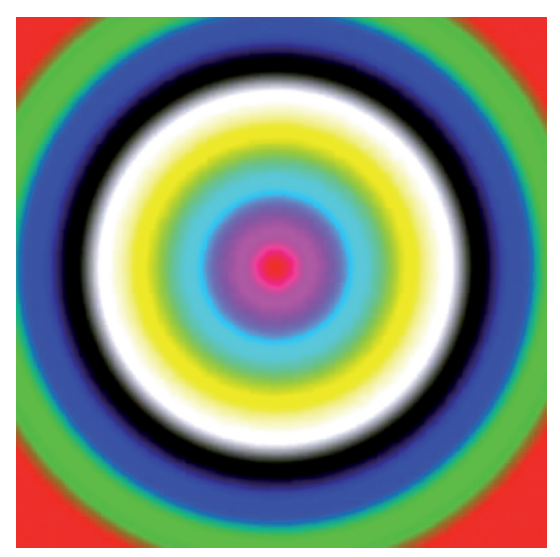

Figura 1. Espiral de colores con movimiento, utilizado para activar el área visual.

\section{Resultados}

La imagen de activación, obtenida con los parámetros descritos anteriormente se corregistra y fusiona con un cerebro Standard MNI 152 (Figuras $2,3,4)$, y con un cerebro que es el resultado del promedio de los T1-3D de los 14 controles utilizados (Figuras 5, 6, 7). 

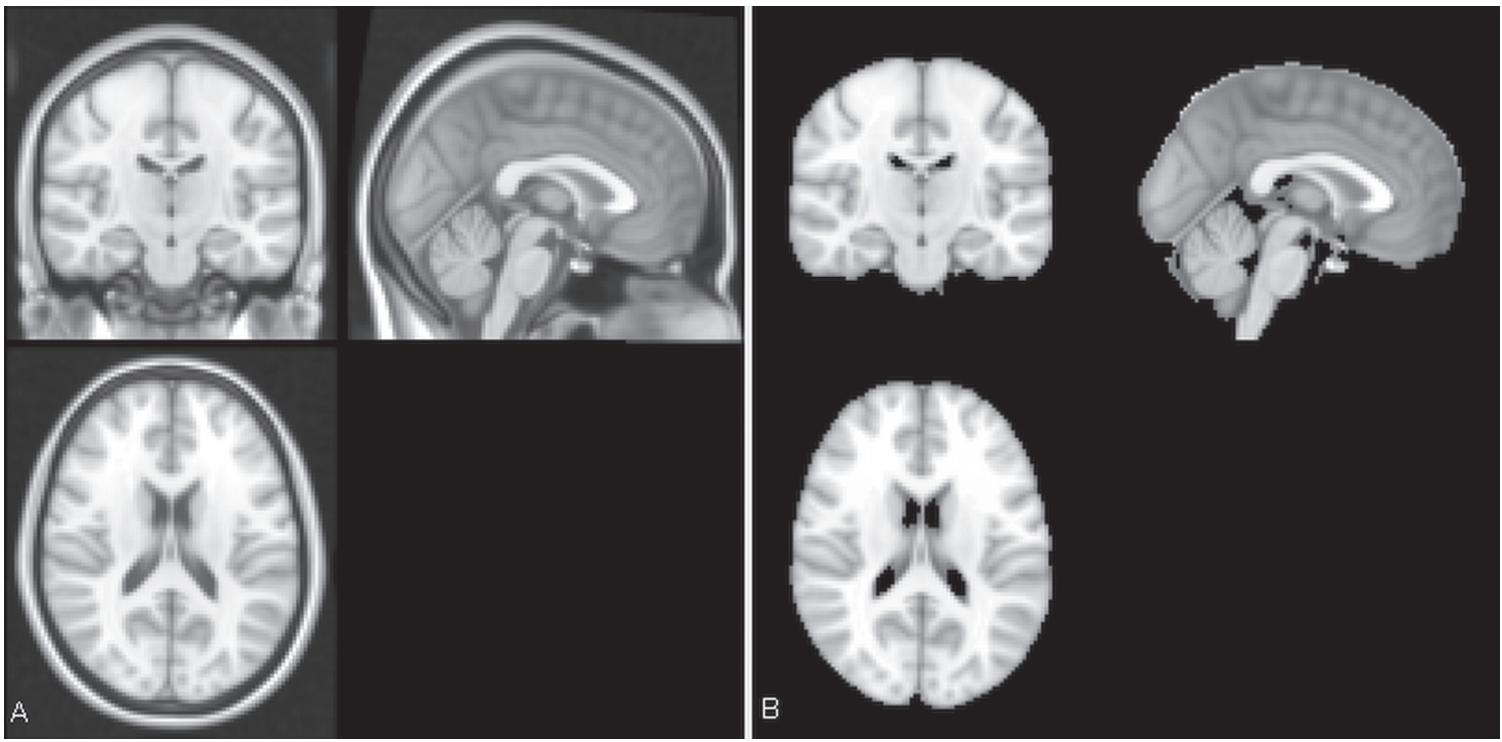

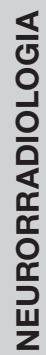

Figura 2. Corte coronal, sagital y axial de la imagen estándar MNI152. a) Imagen con cráneo, y b) Imagen de sustracción de cráneo con software BET (Brain Extraction Tool).

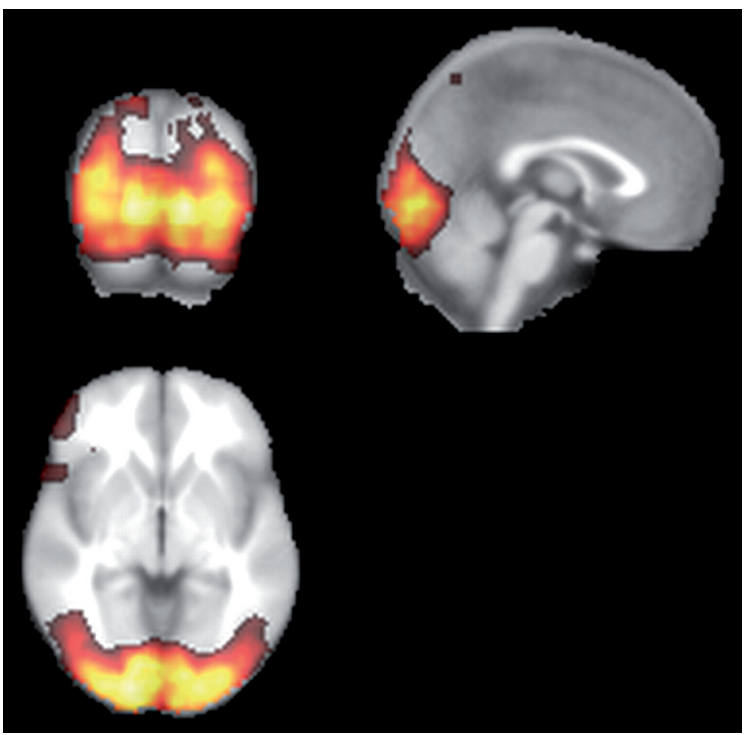

Figura 3. Cortes coronal, sagital y axial del procesamiento de alto nivel de la fMRI visual de los 14 voluntarios. La imagen anatómica T1 utilizada es el Standard MNI152.

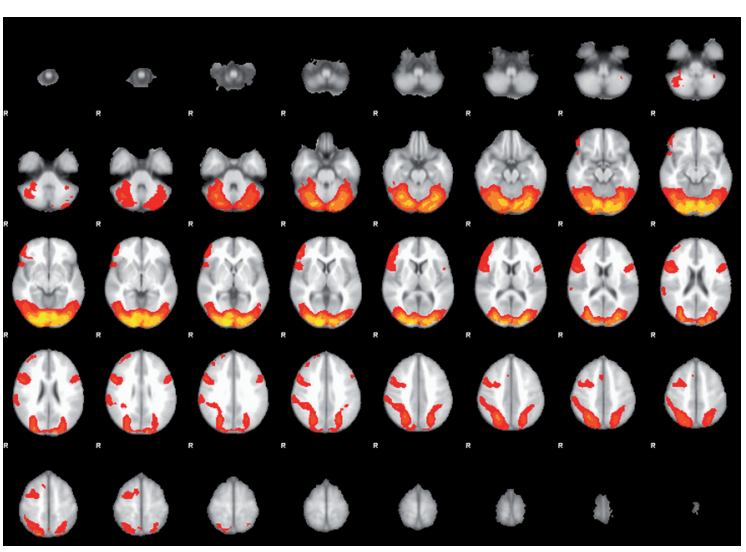

Figura 4. Cortes axiales del procesamiento de alto nivel de la $\mathrm{fMRI}$ visual de los 14 voluntarios. La imagen anatómica T1 utilizada es el Standard MNI152.

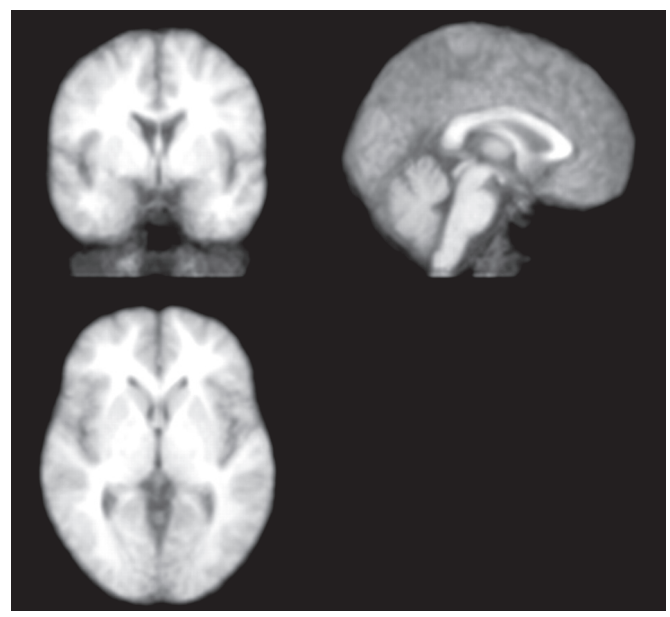

Figura 5. Cortes coronal, sagital y axial de la imagen T1 promedio de los 14 controles, creada con los T1-3D.

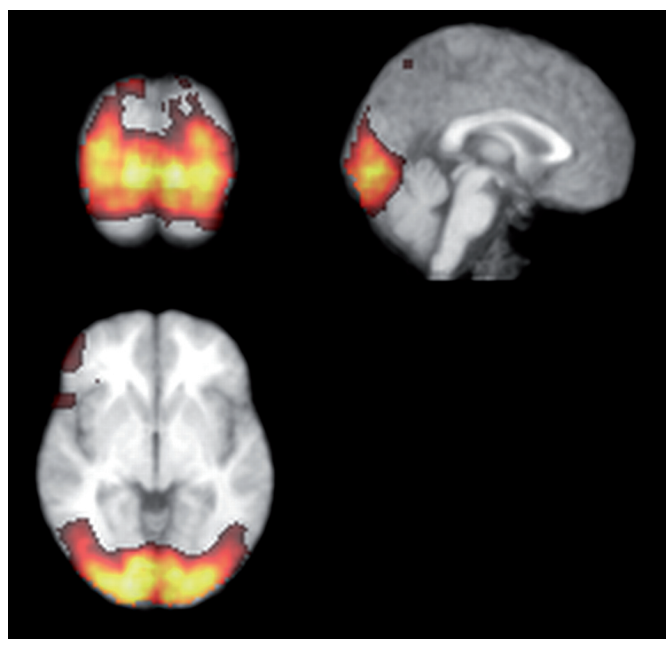

Figura 6. Cortes coronal, sagital y axial del procesamiento de alto nivel de la fMRl visual de los 14 voluntarios. La imagen anatómica T1 utilizada es el promedio de los T1$3 D$ de los 14 controles, Figura 5. 
En Tabla I se incluyen las coordenadas Talairach de los voxeles de mayor activación (con Z>2.7) correspondientes a los tres clusters significativos (Figuras 4,7), además de la región neurológica y el área de Brodmann correspondiente.

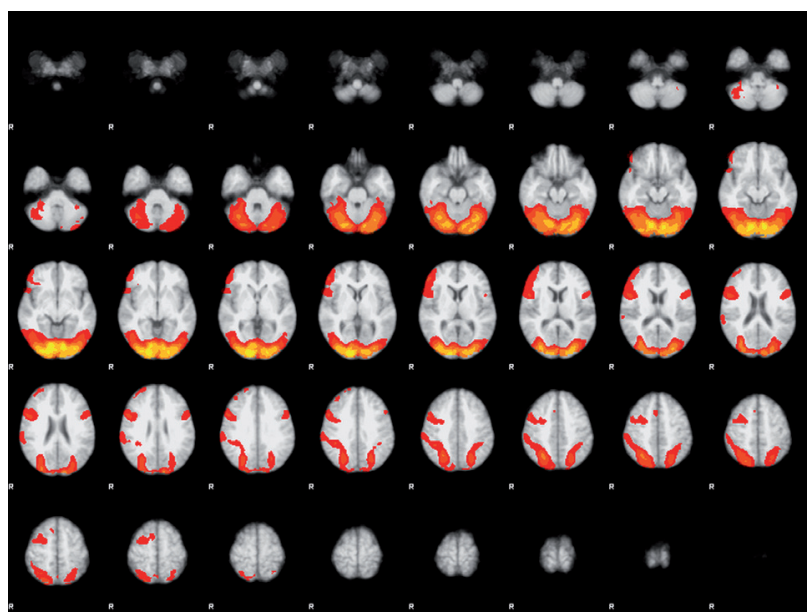

Figura 7. Cortes axiales del procesamiento de alto nivel de la fMRI visual de los 14 voluntarios. La imagen anatómica T1 utilizada es el promedio de los T1-3D de los 14 controles, Figura 5.

\section{Discusión}

Para analizar los resultados obtenidos se debe conocer la anatomía clásica de la vía visual, que incluye: fotorreceptores de las retinas, nervios ópticos, quiasmas, cintillas ópticas, núcleos geniculados laterales y corteza visual. La representación de cada hemicampo visual corresponde a la corteza visual primaria (CVP) contralateral. La CVP se encuentra en el polo posterior del hemisferio, en la región mesial de los lóbulos occipitales adyacente a la cisura calcarina $^{(6,9)}$

Los campos superiores se localizan por debajo de la cisura calcarina y los campos inferiores por encima de ésta. Casi la mitad de esta superficie corresponde a la representación de la fóvea y de la región inmediatamente adyacente a ella, es decir el área de mayor agudeza visual. La CVP (V1) corresponde al área 17 de Brodmann, también llamada corteza estriada porque contiene bandas de sustancia blanca. V1 está ubicada en el polo posterior de la corteza occipital; es altamente especializada en el procesamiento de la información de objetos estáticos y móviles y en el reconocimiento de patrones ${ }^{(9)}$.

En ubicación inmediatamente superior e inferior a la CVP se encuentra el área V2 o corteza visual de asociación (área 18 de Brodmann). Las áreas V1 y V2 son sensibles a la orientación de los estímulos, su color y los contornos de las formas. Existe otra área visual más pequeña (V4) que responde a las formas y se ubica en situación ántero-inferior a $\mathrm{V}^{(6)}$. La información va desde la CVP y secundaria hacia las cortezas temporal y parietal por dos vías diferentes, que además realizan distintas funciones ${ }^{(6)}$. La primera corresponde a la vía ventral o temporal que conecta el área V4 con la región temporal inferior y responde al color, la forma y el reconocimiento de rostros y otras formas complejas; depende de la corteza temporal inferior $^{(6)}$.

Otra vía de información es la dorsal, que a tra-

Tabla I. Coordenadas MNI (standard Montreal Neurological Institute), región neurológica aproximada y área de Brodmann correspondiente de los voxeles de activación de clusters con $Z>2.7$.

\begin{tabular}{|llll}
$\begin{array}{l}\text { Coordenadas } \\
\text { Talairach } \\
\text { x } y\end{array}$ & $\begin{array}{l}\text { Región } \\
\text { neurológica (aprox) }\end{array}$ & Area de Brodmann & Z (cluster) \\
\hline
\end{tabular}

\begin{tabular}{|c|c|c|c|c|c|}
\hline 16 & -93 & 7 & R, Occipital Lobe, Cuneus & 17 & $21.3(1)$ \\
\hline-12 & -82 & -4 & L, Occipital Lobe, Lingual Gyrus & 18 & $20.5(1)$ \\
\hline 12 & -80 & -3 & R, Occipital Lobe, Lingual Gyrus & 18 & $20.3(1)$ \\
\hline 16 & -84 & -3 & R, Occipital Lobe, Lingual Gyrus & 18 & $19.9(1)$ \\
\hline 12 & -78 & 10 & R, Occipital Lobe, Lingual Gyrus & 18 & $18.8(1)$ \\
\hline-18 & -91 & 3 & L, Occipital Lobe, Cuneus & 17 & $18.8(1)$ \\
\hline 52 & 9 & 22 & R, Frontal Lobe, Inferior Frontal Gyrus & 44 & $8.05(2)$ \\
\hline 50 & 9 & 16 & R, Frontal Lobe, Inferior Frontal Gyrus & 44 & $8.04(2)$ \\
\hline 54 & 39 & 4 & R, Frontal Lobe, Inferior Frontal Gyrus & 45 & $7.72(2)$ \\
\hline 55 & 13 & 27 & R, Frontal Lobe, Inferior Frontal Gyrus & 9 & $7.67(2)$ \\
\hline 52 & 10 & 36 & R, Frontal Lobe, Middle Frontal Gyrus & 9 & $6.96(2)$ \\
\hline 59 & 20 & 8 & R, Frontal Lobe, Inferior Frontal Gyrus & 45 & $5.77(2)$ \\
\hline-50 & 12 & 14 & L, Frontal Lobe, Inferior Frontal Gyrus & 44 & $6.53(3)$ \\
\hline-50 & 13 & 20 & L, Frontal Lobe, Inferior Frontal Gyrus & 45 & $6.25(3)$ \\
\hline
\end{tabular}


vés de V2 y V1 se extiende a las áreas temporales medias, temporales súpero-mediales y parietales que intervienen en la representación del mundo visual y en la planificación del movimiento(6). Por lo anterior, el estímulo visual utilizado en los 14 estudios de fMRI realizados a controles debería estimular la mayor cantidad de áreas comprometidas en el proceso visual $^{(6,10,11)}$.

Los clusters correspondientes a las áreas de activación se relacionan con las áreas de Brodmann 17 y 18 (cluster 1 ) y las áreas de Brodmann 9, 44 y 45, que corresponden a los clusters 2 y 3 (Tabla I).

En la imagen resultante (Figura 4), las áreas de activación con $Z>18$ corresponden a los voxeles ubicados en las áreas 17 y 18 de Brodmann (Tabla I) que se relacionan a la CVP (V1) y a la corteza visual de asociación (V2), respectivamente.

Los voxeles con $Z \leq 8.05$ se ubican en las áreas de Brodmann 9, 44 y 45. Estas tres áreas forman parte de la corteza prefrontal dorsolateral (DL-PFC) que es el área cortical responsable de la planificación, organización y regulación motora. Además, tiene un rol importante en la integración tanto de la información sensorial y mnemónica como en la regulación de la acción y función intelectual; también está involucrada en la memoria de trabajo ${ }^{(12-14)}$.

Se demuestra que al realizar una $\mathrm{fMRI}$ visual, se activan principalmente las áreas de la corteza que tienen relación con la percepción y procesamiento visual, es decir, la CVP (V1, área 17 de Brodmann) y la corteza visual de asociación (V2, área 18 de Brodmann).

\section{Agradecimientos}

Damos gracias a todos nuestros controles cuya buena voluntad permitió la realización de este estudio.

\section{Bibliografía}

1. Brett M, Johnsrude IS, Owen AM. The problem of functional localization in the human brain. Nat Rev Neurosci. 2002; 3(3): 243-249.
2. Talairach J, Tournoux P. Co-planar Stereotaxic Atlas of the Human Brain: 3-Dimensional Proportional System - an Approach to Cerebral Imaging. New York, USA. Thieme Medical Publishers, 1988.

3. Talairach J, Tournoux P. Referentially Oriented Cerebral MRI Anatomy: An Atlas of Stereotaxic Anatomical Correlations for Gray and White Matter. New York, USA. Thieme Medical Publishers,1993.

4. Brodmann K. Vergleichende Lokalisationslehre der Grosshirnrinde in ihren Prinzipien dargestellt auf Grund des Zellenbaues, Barth, Leipzig, Germany, 1909.

5. Liney G. MRI from A to Z. A definitive guide for Medical professionals. Primera edición, Edinburgh Building, Cambridge, UK, Cambridge University Press, 2005.

6. Rosales R, Rojas G, Gálvez M, y cols. Obtención de mapas corticales de áreas motoras y visual, con resonancia magnética cerebral funcional. Rev Chil Radiol 2006; 12: 164-169.

7. Barton JJ, Simpson T, Kiriakopoulos E, y cols. Functional $\mathrm{MRI}$ of lateral occipitotemporal cortex during pursuit and motion perception. Ann Neurol 1996; 40: 387-398.

8. Le Bihan D, Turner R, Jezzard P. Activation of human visual cortex by mental representation of visual patterns. Proceedings of the 11 Annual Meeting of the Society of Magnetic Resonance in Medicine, Berlin 1992; $p$ 311.

9. Kandel ER, Schwartz JH, Jessell TM. Principios de neurociencia, 4a edición. Madrid, España, McGrawHill-Interamericana, 2001.

10. Barton JJ, Simpson T, Kiriakopoulos E, y cols. Functional MRI of lateral occipitotemporal cortex during pursuit and motion perception. Ann Neurol 1996; 40: 387-398.

11. Claeys KG, Dupont P, Cornette L, y cols. Color discrimination involves ventral and dorsal stream visual areas. Cereb Cortex 2004; 14: 803-822.

12. Robertson EM, Tormos JM, Maeda F, Pascual-Leone A. The Role of the Dorsolateral Prefrontal Cortex during Sequence Learning is Specific for Spatial Information, Cerebral Cortex 2001; 11: 628-635.

13. Procyk E, Goldman-Rakic PS. Modulation of Dorsolateral Prefrontal Delay Activity during Self-Organized Behavior. J Neurosci. 2006; 26: 11.313-11.323.

14. Pribram KH, Mishkin M, Rosvold HE, Kaplan SJ. Effects of delayed-response performance of lesions of dorsolateral and ventromedial frontal cortex of baboons. J Comp Physiol Psychol 1952; 45: 565-575. 